\title{
DEMOKRATISIERUNG DER EUROPÄISCHEN UNION
}

Claudio Franzius

März 2013

Berliner Arbeitspapier zur Europäischen Integration Nr. 17 Berlin Working Paper on European Integration No. 17 


\section{Inhaltsverzeichnis / Content}

1. Entscheidungsfindung 3

1.1 „Alte“ Institutionen: Kommission, Rat und Gerichtshof 3

1.2 „Neue“ Institutionen: Europäischer Rat (1974), Europäisches Parlament (1979) und Europäische Zentralbank (1993)

1.3 Demokratiedefizit?

2. Maßstab und Methode $\quad 7$

$\begin{array}{lll}2.1 & \text { Zweigliedrige Legitimationsstruktur } & 7\end{array}$

2.2 Von der Gemeinschaftsmethode zur neuen Unionsmethode? 7

2.3 Institutionalisierung der Europäischen Wirtschaftsregierung 8

3. Wege aus der Krise 10

3.1 Demokratische Defizite als funktionale Defizite 10

3.2 Optionen: Direktwahl des Kommissionspräsidenten, Parlamentarisierung des

Regierens oder Reservate mitgliedstaatlichen Handelns? 11

3.3 Große oder kleine Lösungen?

Der Autor / The Author

Claudio Franzius ist Privatdozent an der Freien Universität Berlin und Gastwissenschaftler am Sonderforschungsbereich „Staatlichkeit im Wandel“ an der Universität Bremen. Er vertritt im Sommersemester 2013 eine Professur für Öffentliches Recht an der Universität Bielefeld.

Die Berliner Arbeitspapiere zur Europäischen Integration werden von Prof. Dr. Tanja A. Börzel, Jean Monnet Lehrstuhl für Europäische Integration und Leiterin der Arbeitsstelle Europäische Integration am OttoSuhr-Institut für Politikwissenschaft der Freien Universität Berlin, veröffentlicht. Die Arbeitspapiere sind auf der gemeinsamen Internetseite von Jean Monnet Lehrstuhl und Arbeitsstelle verfügbar: http://www.fuberlin.de/europa

The Berlin Working Papers on European Integration are published by Prof. Dr. Tanja A. Börzel, Jean Monnet Chair for European Integration and Director of the Centre for European Integration of the Otto Suhr Institute for Political Science at the Freie Universität Berlin. The Working Papers are available on the joint website of the Jean Monnet Chair and the Centre: http://www.fu-berlin.de/europa 


\section{Entscheidungsfindung ${ }^{1}$}

\section{1 „Alte“ Institutionen: Kommission, Rat und Gerichtshof}

Wenn wir über die Demokratie in Europa sprechen, müssen wir wissen, wie die Entscheidungen in diesem merkwürdigen Gebilde getroffen werden. Wer trifft die Entscheidungen, um deren Demokratisierung es geht?

Hierfür sollten wir zwischen „alten“ und „neuen“ Institutionen unterscheiden. Die römischen Verträge schufen vor mehr als einem halben Jahrhundert mit der Kommission, dem Rat und dem Gerichtshof ein Dreigestirn, dem wesentliche Aufgaben zugesprochen wurden. Das Herz bildete die Kommission, der als Hüterin des europäischen Gemeinwohls bis heute das Inititativrecht für Rechtssetzungsvorhaben zufällt. Beschlossen werden Rechtsakte vom Ministerrat, der von den Regierungen der Mitgliedstaaten, in denen die Rechtsakte wirksam werden sollen, beschickt wird. Von einem Gesetzgeber war noch nicht die Rede, erschöpfte sich doch die Rechtsetzung auf das Abarbeiten des in den Verträgen niedergelegten Integrationsprogramms, zunächst für Kohle und Stahl, später erweitert um den Binnenmarkt. Nicht zu unterschätzen ist die Rolle des Europäischen Gerichtshofs, der mit seinen Leitentscheidungen van Gend \& Loos und Costa/ENEL vor knapp 50 Jahren die konstitutionellen Grundlagen für die Rechtsgemeinschaft legte. Es galt die Integrität des Rechts vor den Abgründen des Politischen zu bewahren.

\section{2 „Neue“ Institutionen: Europäischer Rat (1974), Europäisches Par- lament (1979) und Europäische Zentralbank (1993)}

Dabei es jedoch nicht geblieben. Die Aufgaben wuchsen, die soziale Dimension des Integrationsprojekts wurde spürbar und im Hintergrund blieb die Idee einer politischen Gemeinschaft präsent. Das institutionelle Dreigestirn hatte sich bewährt, aber zur Bewältigung der neuen Aufgaben traten neue Institutionen zu den alten hinzu. So initierten Valery Giscard d' Estaing und Helmut Schmidt 1974 die Gründung des Europäischen Rates als Impulsgeber für die Europäische Union. In das bisherige Entschei- 
dungsgefüge passte dieses Organ nicht. Augenscheinlich wollten sich die Staats- und Regierungschefs das Zepter in Europafragen nicht aus der Hand nehmen lassen. Der Europäische Rat entwickelte sich zum institutionellen Gegenspieler des ebenfalls neuen, seit 1979 direkt gewählten Europäischen Parlaments, das nach und nach zum europäischen Mitgesetzgeber avancierte, aber der Kommission das Initiativrecht bis heute nicht streitig macht. Immerhin handelt es sich beim Europäischen Parlament um eine einzigartige Einrichtung. Es ist das weltweit stärkste „überstaatliche“ Parlament dieser Art und zeigt, dass der Parlamentarismus nicht an Staaten gebunden ist.

Es gibt neben dem Europäischen Rat und dem Europäischen Parlament noch eine weitere neue Institution, die ins Gerede gekommen ist. Gemeint ist die Europäische Zentralbank, die mit dem Vertrag von Maastricht 1993 geschaffen wurde. Ihre Unabhängigkeit und das Aufgabenprofil, aber auch die Grenzen ihres Tätigwerdens basieren auf dem damals nur schwer zu erzielenden Konsens der Mitgliedstaaten, der vor allem der deutschen Position entgegen kam. Hatte schon der Binnenmarkt eine gefährliche Asymmetrie zwischen die supranationale Markt- und nationaler Sozialpolitik entstehen lassen, so trat mit der Schaffung der Währungsunion keineswegs unbemerkt, sondern politisch gewollt eine weitere Asymmetrie hinzu, denn anders als die Währungspolitik verblieb die Wirtschaftspolitik in der Hand der Mitgliedstaaten. Hier liegt ein Grund für die gegenwärtige Krise und die Suche nach Lösungen. Wer die Strategien, wozu auch die Staatsanleihenankäufe der EZB gehören, pauschal als rechtswidrig brandmarkt, droht zu übersehen, dass wir seit längerer Zeit etwas beobachten können, was gewöhnlich zum Recht dazugehört: Die Wiederkehr der Politik. Europa ist nicht in Stein gemeißelt. Das wirft die Frage auf, wer die Europäische Union politisch voranbringen und demokratisch sichern kann.

\subsection{Demokratiedefizit?}

\subsection{1 „Altes“ Demokratiedefizit und die Antwort: Europäisches Parlament}

Dass wir in Europa ein Demokratiedefizit haben, wurde lange Zeit bestritten. Solange es sich um von den Staaten abgeleitete Herrschaft handelte, die Staaten als „Herren der Verträge" fungierten und sich Europapolitik im Vollzug des in den Verträgen niedergelegten Integrationsprogramms erschöpfte, es sich also um einen Zweckverband funktionaler Integration ohne jeden Anspruch auf Umverteilung handelte - solange 
schien sich die Union demokratische Legitimation von den Staaten gewissermaßen „ausleihen“ zu können.

In dem Maße jedoch, wie die Union dazu überging, eigene, über den Vorrang des Europarechts abgesicherte Herrschaftsgewalt auszuüben, reichte die Legitimation über die gewählten Regierungsvertreter im Rat nicht mehr aus. Sie vertreten die Interessen ihrer Staatsvölker, nicht aber die Gesamtheit der Unionsbürger. Die institutionelle Antwort auf dieses „alte“ Demokratiedefizit war das Europäische Parlament. Es versorgt die Ausübung supranationaler Herrschaft mit demokratischer Legitimation durch die Unionsbürger, nicht die Staatsvölker.

\subsection{2 „Neues“ Demokratiedefizit und der Europäische Rat}

Inzwischen haben wir jedoch ein „neues“ Demokratiedefizit. Das hat mit der Aufwertung des Europäischen Rates als einer verselbständigten internationalen Organisation in der Europapolitik zu tun. Hier werden inzwischen die großen, aber auch eher kleinteilige politische Entscheidungen getroffen.

In dem Maße, wie der Europäische Rat der Aufgabe nachkommt, die nationalen Politiken in einem gemeinsamem Rahmen zu koordinieren und zu integrieren, können sich seine Entscheidungen nicht mehr auf die Legitimation stützen, die sich aus dem demokratischen Charakter seiner Mitglieder herleitet. Denn wir müssen sehen, dass die Reichweite des demokratischen Mandats eines Mitgliedstaates sich nicht mit dem Bereich der gewachsenen Zuständigkeiten des Europäischen Rates deckt. Aus der Sicht der nationalen Wähler bedeutet das Regieren des Europäischen Rates schlicht und ergreifend Fremdbestimmung. Denn aus nationaler Sicht bestimmen fremde Regierungen, welche die Interessen anderer Nationen vertreten, über das eigene politische Schicksal mit, obgleich sie in ihren Entscheidungen nicht an das jeweils eigene demokratische Votum gebunden sind. Hier liegt das grundsätzliche Problem der Verhandlungsdemokratie, in der angesichts der ökonomischen Zwänge zur Kooperation - wie Jürgen Habermas bemerkt - die Neigung wachse, „die Politik des Europäischen Rates 
gegenüber den nationalen Arenen abzuschirmen und dort soweit wie möglich zu dethematisieren". ${ }^{2}$

\subsubsection{Europäische Rolle nationaler Parlamente}

Was tun? Das Europäische Parlament sitzt, wie es dessen Präsident in seiner Kritik am Fiskalvertrag ausdrückt, am Katzentisch. Und der Europäische Rat droht die Legitimationsgrundlagen zu untergraben, auf denen die Union beruht. Wo angesichts der begrenzten Kompetenzausstattung der Unionsorgane nicht supranational, sondern nur intergouvernemental, also zwischenstaatlich gehandelt werden kann, rückt die europäische Rolle der nationalen Parlamente in den Vordergrund.

Dass sie eine Rolle spielen, verdeutlicht der Vertrag von Lissabon mit ihrer ausdrücklichen Anerkennung, etwa mit Blick auf die Subsidiaritätsrüge gegenüber Rechtssetzungsinitiativen der Kommission. Hier geht es jedoch um etwas anderes, nämlich um die Frage, ob darüber zwischenstaatliches Handeln in europäischen Angelegenheiten ausreichend legitimiert werden kann. Ist es möglich, den „Ausfall“ des Europäischen Parlaments als Vertretung der Unionsbürger durch die nationalen Parlamente zu kompensieren? Sie sind es, die aus der Sicht des Bundesverfassungsgerichts Europa mit Legitimation versorgen. Aber das verlangt von den nationalen Parlamenten einen Rollenwandel. Müsste die europäische Rolle der nationalen Parlamente nicht darin bestehen, die von innen kontrollierten Regierungen im Europäischen Rat zu verpflichten, prinzipiell nur solchen Regelungen auf Unionsebene zuzustimmen, die sich gegenüber der Gesamtheit der europäischen Bürgerinnen - also auch den Griechen - rechtfertigen lassen können? Ich möchte es bei dieser Frage belassen, aber Zweifel anmelden, ob eine Demokratisierung der Europäischen Union über die nationale Parlamente wirklich ausreicht.

2 Habermas, J. 2012: Gesichtspunkte für die demokratische Legitimation einer neuen „institutionellen Architektur" der EWU, unveröffentlichte Stellungnahme für die EZB; 6.10.2012. 


\section{Maßstab und Methode}

\subsection{Zweigliedrige Legitimationsstruktur}

Woran bemisst sich das Demokratiedefizit der Europäischen Union? Wir haben gesehen, dass dies eine schwierige Frage ist, die viel mit unseren unterschiedlichen Vorverständnissen zu tun hat und mit dem Problem zu kämpfen hat, dass wir keine Erfahrungen mit der Demokratie eines Gemeinwesens haben, die kein Staat ist, aber auch kein Staat werden will. Immerhin liefert uns der Vertrag von Lissabon einen aufschlussreichen Maßstab. In Art. 10 des reformierten Unionsvertrags heißt es:

„Die Arbeitsweise der Union beruht auf der repräsentativen Demokratie. Die Bürgerinnen und Bürger sind auf Unionsebene unmittelbar im Europäischen Parlament vertreten. Die Mitgliedstaaten werden im Europäischen Rat von inrem jeweiligen Staats- oder Regierungschef und im Rat von ihrer jeweiligen Regierung vertreten, die ihrerseits in demokratischer Weise gegenüber ihrem nationalen Parlament oder gegenüber ihren Bürgerinnen und Bürgern Rechenschaft ablegen müssen."

Aufschlussreich ist diese Formulierung deshalb, weil es für den Normalfall bedeutet: Europäisches Parlament und nationale Parlamente lassen sich nicht gegeneinander ausspielen. Es handelt sich um eine zweigliedrige Legitimationsstruktur.

\subsection{Von der Gemeinschaftsmethode zur neuen Unionsmethode?}

Aber das gilt eben nur für den Normalfall. Nämlich für den Fall, wo das ordentliche Gesetzgebungsverfahren durchgeführt wird und die supranationale Regel der Beteiligung von Europäischem Parlament und Rat greift. Wo demgegenüber der Union keine Kompetenz zugewiesen sind, kann die alte "Gemeinschaftsmethode" mit der Initiative der Kommission und der Entscheidung durch Rat und Parlament auch nicht greifen. Gerade in sensiblen Politikfeldern - wie der Sozialpolitik - halten sich die Mitgliedstaaten zurück, der Union weitere Kompetenzen zu übertragen. Dennoch muss aus ihrer Sicht gehandelt werden. Von der Koordinierung der nationalen Politiken ist die Rede, aber auch von einer "neuen Unionsmethode“. 
Darunter versteht die Bundeskanzlerin ein abgestimmtes solidarisches Handeln - jeder in seiner Zuständigkeit, alle für das gleiche Ziel. Befürchtet wird eine Erosion der Gemeinschaftsmethode, doch sollten wir die Kanzlerin nicht zu sehr schelten. Wo die Gemeinschaftsmethode gar nicht anwendbar ist, weil es an den Kompetenzen fehlt, kann diese auch nicht unterlaufen werden. Das betrifft die Fiskal- und Wirtschaftspolitik, was uns zu der Frage führt, wie sich eine europäische Wirtschaftsregierung demokratisch institutionalisieren ließe.

\subsection{Institutionalisierung der Europäischen Wirtschaftsregierung}

Hier konkurrieren entsprechend den unterschiedlichen Methoden zwei Strategien: Auf der einen Seite haben wir die eben genannte und von den Regierungen bevorzugte „neue“ Unionsmethode, auf der anderen Seite die von den Unionsorganen favorisierte „klassische“ Gemeinschaftsmethode.

Während die Unionsmethode als zwischenstaatliche Variante die Europäische Wirtschaftsregierung in einem neuen Gipfel, nämlich im „Euro-Gipfel“ nach dem Fiskalvertrag, verortet, wählt die Gemeinschaftsmethode als supranationale Variante eine Verortung in den Unionsorganen, namentlich der Kommission mit ihren neuen Überwachungsbefugnissen. Beide Varianten rufen ein Demokratieproblem hervor:

\subsubsection{Flucht in völkerrechtliche Nebenregime: Fiskalvertrag}

Mit dem Fiskalvertrag ist die Europapolitik in ein völkerrechtliches Nebenregime geflüchtet. Das ist zwar rechtlich nicht zu beanstanden, wirft aber die Frage auf, ob die mitgliedstaatliche Legitimation ausreicht, die verstärkte Handlungs- und Steuerungsfähigkeit des Europäischen Rates als demokratisch auszuweisen. Wie sollen die nationalen Parlamente die Festlegung eines finanz- und wirtschaftspolitischen Rahmens ausreichend kontrollieren? Man mag auf die Budgetverantwortung des Deutschen Bundestags und den Erhalt des Nationalstaates setzen, aber der zu schaffende Rahmen für eine faktisch bindende Wirtschaftspolitik steht vor der Herausforderung, dass die jeweils nationalen Legitimationsstränge immer nur die nationale Perspektive auf Europa spiegeln. 
Natürlich macht es Sinn, die Handlungsfähigkeit der europäischen Organe zu stärken. Der Fiskalvertrag - aus der Not geboren - stiftet jedoch keine demokratische Legitimation, sondern privilegiert ein bestimmtes Politikmuster, das sich als wachstumsfeindlich und sozial ungerecht erweist. Einer demokratisch entwurzelten Technokratie - erneut mit den Worten von Jürgen Habermas - fehlen

„sowohl die Macht wie das Motiv, die Forderungen der Wahlbevölkerung nach sozialer Gerechtigkeit, Statussicherheit, öffentlichen Dienstleistungen und kollektiven Gütern im Konfliktfall gegenüber den systemischen Imperativen von Wettbewerbsfähigkeit und Wirtschaftswachstum ausreichend zu berücksichtigen." 3

Insoweit kann nicht alles von den Parlamenten erwartet werden. Zu vermeiden ist, ein Parlamentsoptimismus. Den nationalen Parlamenten bleibt jedoch aufgegeben, den von den Regierungen eingeschlagenen Weg zur Verbesserung einer gemeinsamen Wirtschaftspolitik nicht als alternativlosen Schritt zu „mehr Europa“ auszuweisen, sondern die Europapolitik eindimensionalen Funktionslogiken zu entziehen.

\subsubsection{Kompetenzgrenzen und andere Hürden: Six Pack}

Der Fiskalvertrag - ausgehandelt in den Hinterzimmern zwischenstaatlicher Politik - ist auch weitgehend überflüssig, da er im wesentlichen Regelungen enthält, die sich bereits im so genannten Six Pack finden. Zusammen mit dem Two Pack, das ebenfalls die Zustimmung der konservativ-liberalen Mehrheit des Europäischen Parlaments gefunden hat, haben die Unionsorgane die europäische Wirtschaftsregierung bereits auf den Weg gebracht, im wesentlichen verortet bei der Kommission. Zwar wird mit dem neuen Überwachungsmechanismus für makroökonomische Ungleichgewichte und der verbesserten Sanktionierbarkeit für den Fall der Nichteinhaltung der Vorgaben die Asymmetrie zwischen Währungs- und Wirtschaftspolitik zu mildern versucht. Es bestehen jedoch erhebliche Zweifel, ob diese Änderungen des Rechtsrahmens rechtlich zulässig sind. Solche Zweifel - das an dieser Stelle betont - haben nicht bloß rechts-

3 Habermas, J. 2012: Gesichtspunkte für die demokratische Legitimation einer neuen „institutionellen Architektur" der EWU, unveröffentlichte Stellungnahme für die EZB; 6.10.2012. 
staatliches, sondern auch demokratisches Gewicht. Denn die Einhaltung der Kompetenzgrenzen durch die Unionsorgane ist ein Demokratieschutz zugunsten der Wahrung nationaler Handlungsspielräume. Handlungsspielräume ohne öffentliche Auseinandersetzung durch die „Hintertür“ zu verringern, schmälert das ohnehin nicht mehr einfach zu unterstellende Vertrauen in die europäische Politik.

Es gibt gute Gründe, der Kommission eine zentrale Position in der Koordinierung der nationalen Wirtschaftspolitiken zuzuweisen. Wer sollte das auch sonst machen? Aber alle Vorschläge, die hier ansetzen, ob es ein europäischer Sparkommissar, ein Finanzminister oder eben die Wirtschaftsregierung ist - sie bleiben bedenklich, solange der Frage ausgewichen wird, wem gegenüber dieses Handeln verantwortlich gemacht werden kann. Die Kommission, so wird gesagt, repräsentiere das europäische Gemeinwohl. Entstanden als ein internationales Sekretariat, das sich durch Sachverstand legitimierte, hat die Kommission trotz ihrer geringen Wertschätzung in den nationalen Öffentlichkeiten längst ein Aufgabenprofil erhalten, das einer Regierung im staatlichen Sinne nahekommt. Wir können die Kommission aber solange nicht als Regierung bezeichnen, solange sie für ihr Handeln nicht auch demokratisch verantwortlich gemacht werden kann. Die Bürgerinnen Europas müssen eine Wahl haben, wen sie an der Spitze der Kommission haben wollen. Solange die Europapolitik sich dem Votum der Bürgerinnen nicht stellt, solange wird Europa kaum demokratischer werden können.

\section{Wege aus der Krise}

\subsection{Demokratische Defizite als funktionale Defizite}

Damit sind wir bei den Wegen aus der Krise. Es gehört zu den folgenreichen Irrtümern der Europapolitik, in der Krise auf die Stärkung der Handlungsfähigkeit zu setzen, die Frage nach der Demokratisierung aber auf „bessere“ Zeiten zu verschieben. Richtigerweise ist bereits heute, also bei der Gestaltung der Reform auf die Einbeziehung der transnationalen Öffentlichkeit und der zivilgesellschaftlichen Akteure zu setzen.

Der Zuwachs an Handlungsfähigkeit ist ohne den Zuwachs an demokratischer Legitimation nicht zu haben. Ein Mehr an demokratischer Legitimation kann entgegen landläufiger Vorstellung sehr wohl ein Mehr an Handlungsfähigkeit bedeuten. Um das zu verdeutlichen, muss man sich nur die Krisenpolitik anschauen. Nach der Logik des Fiskalvertrags liegt die Demokratisierung in der „Vergipfelung“ der Politik. Das ist aber 
nicht nur wenig legitim, sondern auch wenig effizient. Demokratische Defizite führen zu funktionalen Defiziten, die mit der Einschränkung von Handlungsmöglichkeiten die Demokratie als Herrschaftsform beschädigen.

Die Krise mag die Stunde der Exekutive sein. Aber das Krisenmangement der letzten Jahre mit immer wieder neuen Gipfeln lässt nationaler Politik kaum noch Zeit und Luft und zum Atmen. Die Krise hat mit erschütternder Klarheit gezeigt, wie wenig der Rückgriff auf die zwischenstaatliche Methode das Versprechen effizienter Krisenbewältigung hat erfüllen können. Durch die Hintertür wurde das Einstimmigkeitsprinzip wieder eingeführt. Einstimmigkeit ermöglicht Blockaden zugunsten nationaler Politik, nicht aber die Ausübung demokratischer Herrschaft im Widerstreit um richtige Lösungen und den Kampf um politische Mehrheiten.

3.2 Optionen: Direktwahl des Kommissionspräsidenten, Parlamentarisierung des Regierens oder Reservate mitgliedstaatlichen Handelns?

Welche Optionen zur Stärkung der Demokratie in Europa gibt es? Die erste Strategie hat ein großer Europäer jüngst wieder ins Spiel gebracht. Gemeint ist Wolfgang Schäuble, der in seiner Karlspreisrede die Direktwahl des Kommissionspräsidenten durch die Unionsbürger vorgeschlagen hat. Gewiss: In Deutschland sträuben sich die Nackenhaare, hatten wir mit dem direkt gewählten Reichspräsidenten nicht ein Problem? Ein Vorteil dieses Vorschlags mag darin liegen, die Ämter des Ratspräsidenten und Kommissionspräsidenten zusammenlegen zu können. Damit würde der Kommissionspräsident im Europäischen Rat gestärkt.

Aus deutscher Sicht liegt freilich eine zweite Strategie näher. Sie besteht in einer stärkeren Rückbindung der „Regierungsspitze“ an das Europäische Parlament. Denn die bisherige Praxis, den Kommissionspräsidenten durch die großen Mitgliedstaaten zu benennen, ist keine gute Lösung. Diese Praxis wäre vielmehr umzukehren und die Wahl der Kommissionsspitze durch das Europäische Parlament zu fordern. Erst wenn die europäischen Parteien mit einem Spitzenkandidaten in den Wahlkampf ziehen, wird es jene Politisierung geben, die es den Bürgerinnen und Bürgern erlaubt, auf die langfristige Ausrichtung der Europapolitik auch Einfluss zu nehmen. Solange der Europawahlkampf demgegenüber den Wählerinnen und Wählern eine Folgenlosigkeit mit 
Blick auf die Kommission signalisiert und diese in der personellen und politischen Ausrichtung nicht stärker an das Wählervotum gebunden wird, bleibt es bei der Dominanz der staatlichen Exekutiven mit der Verlängerung ihrer nationalen Interessen auf das Handeln der Union.

Man mag sich fragen, ob eine Direktwahl des Kommissionspräsidenten unmittelbar durch die Unionsbürger oder mittelbar durch das Europäische Parlament vorzugswürdig ist. Wir sollten uns jedoch bewusst sein, dass es im europäischen Institutionengefüge nach allen Erfahrungen kaum gelingen wird, das eigene Modell ohne Anpassungen auf die Union zu projizieren. Bislang waren es immer Mischformen, in denen Kompromisse zum Ausdruck gekommen sind. Etwa dergestalt, einerseits den Präsidenten des Europäischen Rates zu schaffen, jedoch andererseits das Vorschlagsrecht des Europäischen Rates für den Kommissionspräsidenten an die Berücksichtigung der Ergebnisse der Wahlen zum Europäischen Parlament zu knüpfen. Während erstes einer Präsidialverfassung entgegen kommt, mag zweites die Entwicklung zu einem parlamentarischen Regierungssystem nahelegen. Beides sind jedoch keine einfachen Lösungsangebote für ein politisches Gebilde, in der die Macht nicht in einer Person oder einem Organ konzentriert ist, auf deren Legitimation dann primär zu setzen wäre. So war auch die gestellte Frage, wer den Friedensnobelpreis für die Europäische Union entgegennehmen soll, zu stark von der Außenvertretungsbefugnis eines Staates gedacht. Die Union ist aber kein Staat und sie will es nicht sein. Sie muss es aber auch nicht sein, um demokratischer zu werden.

Deshalb noch ein Wort zu einer dritten Strategie. Sie zielt zum Schutz der mitgliedstaatlichen Demokratie auf das Abstecken von Reservaten staatlichen Handelns. Den Anspruch der Europäischen Union darauf zu beschränken, das Handeln demokratischer Staaten im Kollisionsfall nur auszubalancieren, erscheint mir angesichts der ökonomischen Verflechtungen und politischen Verschränkungen nicht zielführend zu sein. Wir sollten deshalb auch nicht nach politischen Primärräumen suchen, die dem europäischen Zugriff von vornherein entzogen wären. Solche Festschreibungen drohen die Asymmetrien und damit auch das Demokratieproblem zu vertiefen. Richtig aber ist, dass Europa die mitgliedstaatlichen Demokratien befähigen muss, in den nationalen Entscheidungsprozessen die Repräsentation der europäischen Interessen zu gewährleisten und angemessen zu berücksichtigen. 


\subsection{Große oder kleine Lösungen?}

\subsubsection{Konvent?}

Wie sollen die beschriebenen Asymmetrien abgebaut werden, wie der Einsicht des Präsidenten des Bundesverfassungsgerichts entsprochen werden, demzufolge Europa eine Zukunft nur als demokratisch legitimierte Rechtsgemeinschaft habe? Als große Lösung wird die Einberufung eines Konvents gefordert, um die notwendigen Anpassungen im Primärrecht vornehmen zu können. Daran wird kein Weg vorbeiführen, sollte die Wirtschaftsregierung eine vergipfelte Einrichtung des Europäischen Rates bleiben. Dessen Handeln ist weder europäisch noch mitgliedstaatlich demokratisch hinreichend legitimiert.

Ob es die Zustimmung aller Mitgliedstaaten zu den notwendigen Reformen geben wird, ist allerdings fraglich. Hier liegt eine Hürde für die Demokratisierung der Europäischen Union. Denn erforderlich werden Vertragsänderungen, die unter einem doppelten Einstimmigkeitsvorbehalt stehen, zunächst bei der Beschlussfassung und dann bei der Ratifikation nach Maßgabe der Regelungen des Mitgliedstaates. Damit steht der Reformprozess unter erheblichen Risiken, die von den Regierungen zum Anlass genommen werden, andere Lösungen außerhalb des unionsrechtlichen Rahmens vorzuziehen.

Dieses Problem ist nicht einfach zu lösen. Die Verträge lassen sich schwerer ändern als jede Verfassung. Das provoziert die Flucht in Sonderregime und damit die Institutionalisierung einer Politik, die mit gegenläufigen Interessen - man denke etwa an stärker wachstumsorientierte Perspektiven - nur schwer in einen Ausgleich gebracht werden können. An Stelle von großen Lösungen wird es deshalb um kleine Lösungen im Rahmen des geltenden Rechts gehen müssen. Diesem Recht ist wegen seiner erschwerten Abänderbarkeit eine Dynamik und Flexibilität eingebaut, was für die Interpretation der Kompetenzen nicht folgenlos bleiben kann.

\subsubsection{Paritätische Beteiligung von Europäischem Parlament (Unionsbürger) und Rat (Staatsangehörige)}

Zum Schluss sei noch eine „rekonstruktive“ Perspektive angeben. Das, was wir in Europa beobachten können, ist uns aus Deutschland im Grunde ja seit langer Zeit ver- 
traut. Wir haben es mit einem „Exekutivföderalismus“ zu tun. Streit und Konflikt werden hinter die Erwartung von Konsens auch dort gestellt, wo formal mit Mehrheit entschieden werden kann.

Das begünstigt eine Steuerung ohne Gegensteuerung, die, soll sie eine andere Politik möglich machen, ein hohes Maß an Selbstmobilisierung voraussetzt. Deshalb erkennen wir Partizipation und die Rolle zivilgesellschaftlicher Akteure in Europa ausdrücklich als demokratisch an. Dies umso mehr als das Bekenntnis der politischen Eliten zur europäischen Einigung immer weniger trägt und die Öffentlichkeit von technokratischen Plänen für ein demokratisches Europa unbeeindruckt bleibt. Die politisch-kulturellen Selbstverständlichkeiten und damit die Parameter der öffentlichen Diskussion werden sich nicht ganz „ohne die zähe Maulwurfsarbeit sozialer Bewegungen“ (Jürgen Habermas) verschieben lassen.

Worin besteht das Ziel? Das Ziel kann nicht in einem europäischen Wohlfahrtsstaat liegen, soweit dieser ein Ende der nationalen Demokratien bedeuten würde. Die Verschränkung der Ebenen zu einem europäischen Bundesstaat auszubauen wäre keine gute Lösung. Es würde zu einer Einebnung der Wege führen, den die Mitgliedstaaten über Jahrhunderte in der Entwicklung ihrer eigenen Demokratie beschritten haben.

Wenn der Umbau der Kommission zu einer Regierung fortgesetzt wird, sollte eine paritätische Beteiligung von Unionsbürgern und Angehörigen der Staatsvölkern an der Regierungsbildung sichergestellt werden. Dem würde die doppelte Verantwortung der Kommission gegenüber dem Europäischen Parlament einerseits und dem Rat andererseits entsprechen. Beide Organe müssten an der Gesetzgebung paritätisch beteiligt werden, denn für die europäische Demokratie ist die Doppelrolle der Bürger konstitutiv: Sie sind einerseits Unionsbürger, vertreten im Europäischen Parlament. Doch zugleich bleibt jeder Unionsbürger Angehöriger eines europäischen Volkes, das vom Mitgliedstaat im Rat vertreten wird. So gesehen haben die europäischen Bürger mit den Worten von Habermas 
„gute Gründe dafür, dass der jeweils eigene Nationalstaat in der Rolle des Mitgliedstaates weiterhin die konstitutionelle Rolle eines Garanten von Recht und Freiheit spielt." 4

Um die soziale Demokratie zu bewahren, sind die Staaten jedoch gehalten, sie europäisch zu verteidigen. An die Stelle von Abschottung muss Einmischung treten. Denn lassen wir uns nicht täuschen: Nur über demokratische Entscheidungsstrukturen wird es möglich sein, die Handlungsfähigkeit auf Unionsebene auszubauen und auf nationaler Ebene zu erhalten.

${ }^{4}$ Habermas, J. 2011: Zur Verfassung Europas: Ein Essay, Berlin, 39.; siehe dazu Franzius, C. 2012 : Buchbesprechung: Jürgen Habermas, Zur Verfassung Europas, in: European Law Journal 18: 6, 887-891. 


\section{Berliner Arbeitspapiere zur Europäischen Integration Berlin Working Paper on European Integration}

1 Tanja A. Börzel: European Governance. Verhandlungen und Wettbewerb im Schatten der Hierarchie, April 2007

2 Franziska Süllke: Young People and Active European Citizenship. Strengthening Opportunities for Citizenship Education on a Local Level. Examples from Practice under Difficult Conditions, Mai 2007

3 Sabine von Oppeln: Das Europäische Sozialmodell. Bilanz und Perspektiven, September 2007

4 Diana Panke: Why the ECJ Restores Compliance Faster in Some Cases Than in Others. Comparing Germany and the UK, Oktober 2007

5 Osvaldo Saldías: Supranational Courts as Engines of Disintegration. The Case of the Andean Community, November 2007

6 Jana Katharina Grabowsky: Mehr als Wirtschaft. Der Beitrag des Airbusprojekts zur europäischen Identität, November 2007

7 Tanja A. Börzel, Yasemin Pamuk, Andreas Stahn: Good Governance in the European Union, Januar 2008

8 Martin Humburg: The Open Method of Coordination and Integration. The Example of European Education Policy, Juni 2008

9 Vera van Hüllen: Transnationalising Euro-Mediterranean Relations. The EuroMediterranean Human Rights Network as an Intermediary Actor, November 2008

10 Claudio Franzius: Warum Governance?, Februar 2009

11 Tanja A. Börzel and Thomas Risse: Venus Approaching Mars? The European Union as an Emerging Civilian World Power, April 2009

12 Julian Schwartzkopff: Splendid Isolation? The Influence of Interest Groups on EU Trade Policy, August 2009

13 Tanja A. Börzel, Tobias Hofmann, Diana Panke: Opinions, Referrals, and Judgments: Analyzing Longitudinal Patterns of Non-Compliance, September 2009 
14 Avram, Andrei: Promoting Good Governance in the Republic of Moldova: The Approach of the European Union, March 2010

15 Avram, Andrei: Promoting Good Governance in the Republic of Moldova: The Approach of the European Union, March 2010

16 Tanja A. Börzel: EU-Staatlichkeit - wie viel und wozu?, März 2013

17 Claudio Franzius: Demokratisierung der Europäischen Union, März 2013 